\title{
Schwartz 函数的分解及其应用
}

\author{
魏明权 ${ }^{(1)}$ ，石坐顺华 ${ }^{(2)}$ ，燕敦验 ${ }^{(1) * ~}$ \\ (1) 中国科学院大学数学科学学院, 北京 100190 ; \\ (2) 中国科学院数学与系统科学研究院数学研究所, 北京 100190 \\ E-mail: weimingquan11@mails.ucas.ac.cn, shizuoshunhua11b@mails.ucas.ac.cn,ydunyan@ucas.ac.cn \\ 收稿日期: 2014-11-21；接受日期: 2015-01-21；网络出版日期: 2015-12-01；＊通信作者 \\ 国家自然科学基金 (批准号: 11471039 和 11271162) 资助项目
}

摘要 本文主要研究 Schwartz 空间 (记作 $\mathscr{S}\left(\mathbb{R}^{n}\right)$ ) 上的函数及 $\mathscr{D}\left(\mathbb{R}^{n}\right)$ 空间上的函数分解问题. 本文用 构造性的方法自适应地证明，任何一个 Schwartz 函数都可以分解为两个 Schwartz 函数的乘积. 进一步, 用类似的方法也证明了每一个 $\mathscr{D}\left(\mathbb{R}^{n}\right)$ 函数都可以分解为与其支集完全相同的两个 $\mathscr{D}\left(\mathbb{R}^{n}\right)$ 函数的乘 积. 作为这两空间上函数分解的应用, 很容易得到 $\mathscr{S}\left(\mathbb{R}^{n}\right)=\mathscr{S}\left(\mathbb{R}^{n}\right) \mathscr{S}\left(\mathbb{R}^{n}\right)$ 和 $\mathscr{D}\left(\mathbb{R}^{n}\right)=\mathscr{D}\left(\mathbb{R}^{n}\right) \mathscr{D}\left(\mathbb{R}^{n}\right)$.

关键词 Schwartz 函数 $\mathscr{D}\left(\mathbb{R}^{n}\right)$ 函数 Fourier 变换 紧支集

MSC (2010) 主题分类 $42 \mathrm{~B} 15,42 \mathrm{~B} 35$

\section{1 引言}

我们知道, Schwartz 函数在调和分析和偏微分方程中起着重要作用, Schwartz 函数空间定义为

$$
\mathscr{S}\left(\mathbb{R}^{n}\right):=\left\{\phi: \phi \in C^{\infty}\left(\mathbb{R}^{n}\right), \forall \alpha, \beta \in \mathbb{N}_{0}^{n}, \rho_{\alpha, \beta}(\phi)=\sup _{x \in \mathbb{R}^{n}}\left|x^{\alpha} D^{\beta} \phi(x)\right|<\infty\right\},
$$

其中 $\mathbb{N}_{0}=\{0,1,2, \ldots\}, \mathbb{N}_{0}^{n}=\mathbb{N}_{0} \times \mathbb{N}_{0} \times \cdots \times \mathbb{N}_{0}$.

由这个定义, 我们很容易看出两个 Schwartz 函数的乘积依然是 Schwartz 函数. 众所周知, Fourier 变换是从 Schwartz 函数空间到自身的一个同胚映射, 因此, 不难得出两个 Schwartz 函数的卷积也 是 Schwartz 函数. 我们可以简单地表示为 $\mathscr{S}\left(\mathbb{R}^{n}\right) \mathscr{S}\left(\mathbb{R}^{n}\right) \subset \mathscr{S}\left(\mathbb{R}^{n}\right), \mathscr{S}\left(\mathbb{R}^{n}\right) * \mathscr{S}\left(\mathbb{R}^{n}\right) \subset \mathscr{S}\left(\mathbb{R}^{n}\right)$. 由 Schwartz 函数的定义, 显然, 可以得出 $1 \notin \mathscr{S}\left(\mathbb{R}^{n}\right)$, 所以, 不容易看出 $\mathscr{S}\left(\mathbb{R}^{n}\right) \mathscr{S}\left(\mathbb{R}^{n}\right) \supset \mathscr{S}\left(\mathbb{R}^{n}\right)$ 一定 成立.

Kamiński ${ }^{[1]}$ 提出了这一经典的分析问题, 即 $\mathscr{S}\left(\mathbb{R}^{n}\right) \mathscr{S}\left(\mathbb{R}^{n}\right) \supset \mathscr{S}\left(\mathbb{R}^{n}\right)$ 是否成立? 很庆幸的是, 文 献 $[2,3]$ 用纯代数的方法证明了这个结论是成立的, 其证明思想可粗略地表示如下: 将 $\mathscr{S}\left(\mathbb{R}^{n}\right)$ 看成 是一个乘法群, 利用代数学中乘法群的一个基本原理可证明 $\mathscr{S}\left(\mathbb{R}^{n}\right)$ 中的每一个元素在这个群中都可 分解为其他两个元素的乘积. 因此, 根据文献 $[2,3]$ 中的结论可知, 对于 $\varphi \in \mathscr{S}\left(\mathbb{R}^{n}\right)$, 存在两个函数 $\varphi_{1} \in \mathscr{S}\left(\mathbb{R}^{n}\right)$ 和 $\varphi_{2} \in \mathscr{S}\left(\mathbb{R}^{n}\right)$, 使得 $\varphi=\varphi_{1} \varphi_{2}$ 成立. 然而, 通过文献 [2,3] 中的方法, 只能得出分解的存 在性, 却不能明确给出 $\varphi_{1}$ 和 $\varphi_{2}$ 的具体表达形式. 更进一步地, 也无法给出 $\varphi_{1}$ 和 $\varphi_{2}$ 对 $\varphi$ 的依赖关 
系. 有关这一问题的研究, 目前还没有实质性的成果. 事实上, 要得出这种依赖关系, 单纯用代数方法 是不够的. 这是一个经典的分析问题, 我们认为应该用分析的方法对 Schwartz 函数的性质作更深入研 究. 为此, 我们很自然地提出了如下问题: 对任意一个给定的 $\varphi \in \mathscr{S}\left(\mathbb{R}^{n}\right)$, 能否给出分解式中 $\varphi_{1}$ 和 $\varphi_{2}$ 更精确的表达形式? 能否明确地给出 $\varphi_{1}$ 和 $\varphi_{2}$ 对 $\varphi$ 的依赖关系?

本文将使用纯分析的方法和构造性证明的技巧对以上提出的两个问题给出肯定的回答. 对 $\mathscr{D}\left(\mathbb{R}^{n}\right)$ 函数, 我们也能得出类似的回答: 对任何一个函数 $\phi \in \mathscr{D}\left(\mathbb{R}^{n}\right)$, 我们可以找到相应的两个函数 $\phi_{1} \in$ $\mathscr{D}\left(\mathbb{R}^{n}\right)$ 和 $\phi_{2} \in \mathscr{D}\left(\mathbb{R}^{n}\right)$, 使得 $\phi=\phi_{1} \phi_{2}$, 并且 $\operatorname{supp} \phi=\operatorname{supp} \phi_{1}=\operatorname{supp} \phi_{2}$. 作为这两个分解的应用, 我 们可以很容易得出 $\mathscr{S}\left(\mathbb{R}^{n}\right)=\mathscr{S}\left(\mathbb{R}^{n}\right) \mathscr{S}\left(\mathbb{R}^{n}\right), \mathscr{D}\left(\mathbb{R}^{n}\right)=\mathscr{D}\left(\mathbb{R}^{n}\right) \mathscr{D}\left(\mathbb{R}^{n}\right)$ 这两个结论. 需要强调的是, 本文 利用了自适应的思想和方法, 即 $\varphi_{1}$ 和 $\phi_{1}$ 分别自适应地依赖于 $\varphi$ 和 $\phi$.

本文的主要定理表述如下:

定理 1 每个 Schwartz 函数都可以表示成两个 Schwartz 函数的乘积.

定理 2 若 $\Phi \in \mathscr{D}\left(\mathbb{R}^{n}\right)$, 则存在两个函数 $\Psi \in \mathscr{D}\left(\mathbb{R}^{n}\right)$ 和 $\Upsilon \in \mathscr{D}\left(\mathbb{R}^{n}\right)$ 使得

$$
\Phi=\Psi \Upsilon
$$

并且

$$
\operatorname{supp} \Phi=\operatorname{supp} \Psi=\operatorname{supp} \Upsilon .
$$

在 $\mathscr{D}\left(\mathbb{R}^{n}\right)$ 函数分解的证明过程中, 我们将反复使用支集的边界等相关定义及符号, 在此统一约定 如下: 函数 $\Phi \in \mathscr{D}\left(\mathbb{R}^{n}\right)$ 的支集、支集的内部和支集的边界分别为 $C, \Omega$ 和 $\partial C$, 即

$$
C=\operatorname{supp} \Phi=\overline{\left\{x \in \mathbb{R}^{n}: \Phi(x) \neq 0\right\}}, \quad \Omega=C^{\circ}, \quad \partial C=C \backslash \Omega .
$$

显然, 当 $\Phi \in \mathscr{D}\left(\mathbb{R}^{n}\right)$ 并且 $\Phi \neq 0$ 时, $\Omega$ 是 $\mathbb{R}^{n}$ 中的一个开集, 边界 $\partial C$ 为紧集. 同时, 我们记 $\mathbb{R}^{n}$ 中任 意一点 $x$ 到边界 $\partial C=C \backslash \Omega$ 的距离 $d_{x}$ 定义为

$$
d_{x}=d(x, \partial C)=\inf _{y \in \partial C}|x-y|
$$

\section{2 主要引理}

为了定理证明的需要, 先给出几个重要引理. 首先用构造性的方法给出一个待定 Schwartz 函数. 选取一个函数 $\varphi \in \mathscr{D}\left(\mathbb{R}^{n}\right)$ 满足 $0 \leqslant \varphi \leqslant 1$, 并且

$$
\varphi(x)= \begin{cases}1, & |x| \leqslant 1, \\ 0, & |x| \geqslant 2 .\end{cases}
$$

由 (2.1) 中 $\varphi$ 的定义, 对所有的 $x \in \mathbb{R}^{n}$, 可得

$$
\varphi(x)+\sum_{j=1}^{\infty}\left(\varphi\left(2^{-j} x\right)-\varphi\left(2^{-j+1} x\right)\right)=1 .
$$

令

$$
\phi(x)=\varphi(x)-\varphi(2 x)
$$


则可以把 $(2.2)$ 简记为

$$
\varphi(x)+\sum_{j=1}^{\infty} \phi\left(2^{-j} x\right)=1 .
$$

选取一个单调上升正数序列 $\left\{\lambda_{j}\right\}_{j} \geqslant 1$, 满足当 $j \rightarrow \infty$ 时, $\lambda_{j} \rightarrow \infty$. 借助于序列 $\left\{\lambda_{j}\right\}$, 由 (2.4) 来 构造函数 $\Psi$ :

$$
\Psi(x):=\varphi(x)+\sum_{j=1}^{\infty} 2^{-\lambda_{j} j} \phi\left(2^{-j} x\right) .
$$

由 (2.4) 所定义的函数 $\Psi$ 与 (2.3) 有明显的区别, 函数 $\Psi$ 是对 $(2.3)$ 左边级数中各项乘以系数 $2^{-\lambda_{j} j}$ 得到的. 随着 $j$ 的增大, $2^{-\lambda_{j} j}$ 迅速趋于 0 , 因此, 函数 $\Psi$ 在 $\infty$ 处具有强烈的衰减性, 而且满足 $0<\Psi(x) \leqslant 1$.

下面来讨论函数 $\Psi$ 的性质. 显然, 当 $|x| \rightarrow \infty$ 时, $\Psi(x) \rightarrow 0$. 进一步, 我们还可得到如下两个结果:

引理 3 设 $\varphi, \phi,\left\{\lambda_{j}\right\}_{j \geqslant 1}$ 和 $\Psi$ 如上定义, 则当 $2^{k} \leqslant|x|<2^{k+1}$ 时, 有

$$
2^{-(k+1) \lambda_{k+1}} \leqslant \Psi(x) \leqslant 2^{-(k-1) \lambda_{k-1}} .
$$

证明 令 $2^{k} \leqslant|x|<2^{k+1}$, 只需考虑 $k \geqslant 2$ 的情形. 由 $\Psi, \varphi$ 和 $\phi$ 的定义, 可以看出

$$
\Psi(x)=\sum_{j=k-1}^{k+1} 2^{-\lambda_{j} j} \phi\left(2^{-j} x\right) .
$$

注意到 $\left\{\lambda_{j}\right\}$ 是单调上升的, 通过简单的不等式放缩, 我们得到

$$
\Psi(x) \leqslant 2^{-(k-1) \lambda_{k-1}} \sum_{j=k-1}^{k+1} \phi\left(2^{-j} x\right)=2^{-(k-1) \lambda_{k-1}},
$$

并且

$$
\Psi(x) \geqslant 2^{-(k+1) \lambda_{k+1}} \sum_{j=k-1}^{k+1} \phi\left(2^{-j} x\right)=2^{-(k+1) \lambda_{k+1}} .
$$

在上面的放缩中, 我们用到了 $\Psi$ 的支集包含于圆环 $\left\{x \in \mathbb{R}^{n}: \frac{1}{2} \leqslant|x| \leqslant 2\right\}$ 这个简单事实.

实际上, 引理 3 给出了 $\Psi$ 的局部大小的估计, 下面这个引理将能给出 $\Psi$ 在将刻画无穷远处的下 降速度.

引理 4 设 $\varphi, \phi$ 和 $\left\{\lambda_{j}\right\}_{j \geqslant 1}$ 如上定义, 并且 $\Psi$ 满足 $(2.4)$, 则 $\Psi \in \mathscr{S}\left(\mathbb{R}^{n}\right)$.

证明 由 Schwartz 函数的定义, 对任意给定的自然数 $N$, 及所有满足 $|\alpha| \leqslant N$ 的多重指标 $\alpha$, 只 需要证明

$$
\lim _{|x| \rightarrow \infty}\left(1+|x|^{2}\right)^{\frac{N}{2}}\left|D^{\alpha} \Psi(x)\right|=0
$$

成立即可. 设 $x \in \mathbb{R}^{n}$ 并且 $|x|$ 充分大, 那么必然存在一个自然数 $l$ 满足 $2^{l} \leqslant|x|<2^{l+1}$. 这样我们可以 得出

$$
\begin{aligned}
\left(1+|x|^{2}\right)^{\frac{N}{2}}\left|D^{\alpha} \Psi(x)\right| & =\left(1+|x|^{2}\right)^{\frac{N}{2}}\left|\sum_{j=l-1}^{l+1} D^{\alpha}\left(2^{-j \lambda_{j}} \phi\left(2^{-j} x\right)\right)\right| \\
& \leqslant\left(1+|x|^{2}\right)^{\frac{N}{2}}\left(\sum_{j=l-1}^{l+1} 2^{-j \lambda_{j}}\left|D^{\alpha}\left(\phi\left(2^{-j} x\right)\right)\right|\right)
\end{aligned}
$$




$$
\leqslant C\left\|D^{\alpha} \phi\right\|_{\infty} 2^{(l+1) N} 2^{-(l-1)\left(\lambda_{l-1}+|\alpha|\right)},
$$

其中 $C$ 是与 $\Psi, \phi$ 和 $N$ 无关的常数.

对于固定的自然数 $N$, 注意到 $2^{l} \leqslant|x|<2^{l+1}$, 所以, 当 $|x| \rightarrow \infty$ 时, $l \rightarrow \infty$, 因此, $\lambda_{l} \rightarrow \infty$. 根据 以上分析, (2.5) 显然成立. 这样我们就证明了引理 4 .

在引理 4 中, 我们给出了一个依赖于序列的 $\left\{\lambda_{j}\right\}_{j \geqslant 1}$ 的 Schwartz 函数 $\Psi$, 这将在证明 Schwartz 函 数分解的过程中起到重要作用, 在后面定理的证明过程中, 我们将看到当 $\left\{\lambda_{j}\right\}_{j \geqslant 1}$ 取合适的序列时, 我 们可以把这里的函数 $\Psi$ 当作分解式中的一个因子. 然而对 $\mathscr{D}\left(\mathbb{R}^{n}\right)$ 的函数, 则需要考虑它在边界 $\partial C$ 处 的下降速度, 类似地构造出一个 $\mathscr{D}\left(\mathbb{R}^{n}\right)$ 函数, 这样通过对待定指标的合适选取, 我们也能得到 $\mathscr{D}\left(\mathbb{R}^{n}\right)$ 函数分解式中的一个因子.

首先, 对任意的 $\mathscr{D}\left(\mathbb{R}^{n}\right)$ 函数, 我们都能得到它在边界处的下降速度.

引理 5 令 $\Phi \in \mathscr{D}\left(\mathbb{R}^{n}\right), x \in \Omega$, 那么, 对任意固定的正整数 $N$,

$$
\lim _{d_{x} \rightarrow 0} d_{x}^{-N}\left(\sum_{|\alpha| \leqslant N}\left|D^{\alpha} \Phi(x)\right|\right)=0
$$

成立.

证明 显然, 边界 $\partial C$ 是一个紧集, 所以, 对给定的 $x \in \Omega$, 必存在一点 $y \in \partial C$ 满足

$$
d_{x}=|x-y|
$$

由 Taylor 展开, 我们得到

$$
\Phi(x)=\Phi(y)+\sum_{k=1}^{N} \frac{1}{k !}[(x-y) \cdot \nabla]^{k} \Phi(y)+\frac{1}{N !} \int_{0}^{1}(1-t)^{N}[(x-y) \cdot \nabla]^{N+1} \Phi(y+t(x-y)) d t .
$$

注意到 $\Phi \in \mathscr{D}\left(\mathbb{R}^{n}\right)$, 且 $C=\operatorname{supp} \Phi$, 由于 $y \in \partial C$, 所以, 对任意多重指标 $\alpha$, 可以得出

$$
D^{\alpha} \Phi(y)=0
$$

把 (2.8) 代入 (2.7) 的 Taylor 展开中, 可以立即得出

$$
\Phi(x)=\frac{1}{N !} \int_{0}^{1}(1-t)^{N}[(x-y) \cdot \nabla]^{N+1} \Phi(y+t(x-y)) d t .
$$

类似地, 用 $D^{\alpha} \Phi$ 来替换 (2.9) 中的 $\Phi$, 对任意的 $|\alpha| \leqslant N$, 可以得到

$$
D^{\alpha} \Phi(x)=\frac{1}{N !} \int_{0}^{1}(1-t)^{N}[(x-y) \cdot \nabla]^{N+1}\left(D^{\alpha} \Phi\right)(y+t(x-y)) d t .
$$

记

$$
M_{k}=\max _{|\beta| \leqslant k}\left\|D^{\beta} \Phi\right\|_{\infty} .
$$

因此, 根据 (2.10), 对所有的 $|\alpha| \leqslant N$, 简单计算可以得出

$$
\left|D^{\alpha} \Phi(x)\right| \leqslant C_{N+1} M_{2 N+1} d_{x}^{N+1} .
$$

由此可以等价地表示为

$$
d_{x}^{-N}\left|D^{\alpha} \Phi(x)\right| \leqslant C_{N+1} M_{2 N+1} d_{x}
$$


对不等式 (2.11) 中对所有满足的 $|\alpha| \leqslant N$ 的 $\alpha$ 求和, 可得到

$$
\lim _{d_{x} \rightarrow 0} d_{x}^{-N} \sum_{|\alpha| \leqslant N}\left|D^{\alpha} \Phi(x)\right|=\lim _{d_{x} \rightarrow 0} C_{\alpha} C_{N+1} M_{2 N+1} d_{x}=0 .
$$

这样我们就完成了引理 5 的证明.

下面运用单位分解的思想来构造一个含有待定指标的 $\mathscr{D}\left(\mathbb{R}^{n}\right)$ 函数, 并且它的支集也为 $C$.

在引言中, 我们提到 $\Omega$ 是一个开集, 这样运用开集的 Whitney 分解 ${ }^{[4-7]}$, 可以找到一列不相交的 可数方体 $\left\{Q_{j}\right\}_{j=1}^{\infty}$ 满足:

(1) $\Omega=\bigcup_{j=1}^{\infty} Q_{j}$;

(2) $Q_{i}^{o} \cap Q_{j}^{o}=\emptyset$, 若 $i \neq j$;

(3) $C_{1} \operatorname{diam}\left(Q_{j}\right) \leqslant d_{x_{j}} \leqslant C_{2} \operatorname{diam}\left(Q_{j}\right)$,

其中 $C_{1}$ 和 $C_{2}$ 是两个常数, $x_{j}$ 表示方体 $Q_{j}$ 的中心.

记 $Q$ 为中心在原点, 边长为 1 的单位方体. 对充分小的正数 $\varepsilon>0$, 选取一个合适的 $\varphi \in \mathscr{D}\left(\mathbb{R}^{n}\right)$ 满足如下性质:

(i) $0 \leqslant \varphi \leqslant 1$;

(ii) $\varphi(x)=1$, 若 $x \in Q$;

(iii) $\varphi(x)=0$, 若 $x \notin(1+\varepsilon) Q$.

定义

$$
\varphi_{j}(x)= \begin{cases}\frac{\varphi\left(\frac{x-c_{j}}{l_{j}}\right)}{\sum_{k=1}^{\infty} \varphi\left(\frac{x-c_{k}}{l_{k}}\right)}, & x \in \Omega, \\ 0, & x \notin \Omega,\end{cases}
$$

其中 $c_{k}$ 和 $l_{k}$ 分别表示方体 $Q_{k}$ 的中心和边长. 显然, $\varphi_{j}$ 的定义是有意义的. 因为由 Whitney 分解 的性质容易看出, (2.13) 右边中分母的求和是有限的; 并且当 $x \in \Omega$ 时, 至少存在一个方体 $Q_{j}$, 使得 $Q_{j} \ni x$, 这样就有

$$
\sum_{k=1}^{\infty} \varphi\left(\frac{x-c_{k}}{l_{k}}\right) \geqslant 1
$$

下面选取一个可数序列 $\left\{\lambda_{j}\right\}_{j=1}^{\infty}$, 使得当 $l_{j} \rightarrow 0$ 时, $\lambda_{j} \rightarrow \infty$. 为了方便起见, 我们不妨假设当 $l_{i} \approx l_{j}$ 时, 满足 $\lambda_{i} \approx \lambda_{j}$.

定义一个依赖于序列 $\left\{\lambda_{j}\right\}_{j=1}^{\infty}$ 的函数 $\Psi$ 为

$$
\Psi(x):=\sum_{j=1}^{\infty}\left(1+\frac{1}{l_{j}}\right)^{-\lambda_{j}} \varphi_{j}(x) .
$$

与引理 4 类似, 我们可以得到如下引理:

引理 6 假设 $l_{j}, c_{j},\left\{\lambda_{j}\right\}_{j=1}^{\infty}, \varphi_{j}$ 和 $\Psi$ 如 (2.13) 和 (2.14) 所定义, 则下面的 3 个结论成立,

(1) 当 $x \in \Omega$ 时, 有 $0<\Psi(x) \leqslant 1$;

(2) 若 $x \in \Omega$, 并且 $2^{-k} \leqslant d_{x}<2^{-k+1}$, 则可以得到 $\Psi(x) \approx 2^{-k \lambda_{k}} \approx d_{x}^{\lambda_{k}}$;

(3) $\Psi \in \mathscr{D}\left(\mathbb{R}^{n}\right)$, 且 $\Omega$ 恰好是 $\Psi$ 的支集的内部.

证明 根据 $\varphi_{j}$ 和 $\Psi$ 在 (2.13) 和 (2.14) 中的定义, 对任意的 $x \in \Omega$, 很容易得出 $0<\Psi(x) \leqslant 1$.

根据我们之前的假设, 当 $l_{i} \approx l_{j}$ 时, 有 $\lambda_{i} \approx \lambda_{j}$. 由 Whitney 分解中 $\left\{Q_{j}\right\}$ 所满足的的性质 (3), 可 以看出, 如果 $x \in \Omega$ 并且满足 $2^{-k} \leqslant d_{x}<2^{-k+1}$, 那么最多存在有限个 $\left\{(1+\varepsilon) Q_{j}\right\}$ 包含 $x$, 同时这些 
方体的边长约等于 $2^{-k}$ 的常数倍. 这样通过 $\Psi$ 的定义, 可以利用类似于引理 3 的方法得到

$$
\Psi(x) \approx 2^{-k \lambda_{k}} \approx d_{x}^{\lambda_{k}} .
$$

引理 $6(3)$ 是我们最关心的, 下面来重点证明这一结果.

首先来证明 $\Psi$ 具有紧支集. 由 $\Omega$ 的分解以及 $\varphi$ 和 $\varphi_{j}$ 在 (2.13) 中的定义, 可以得到, 若 $x \notin \Omega$, 则显然有 $\varphi_{j}(x)=0$, 因此, $\Psi(x)=0$, 这就说明了 $\Psi$ 具有紧支集, 并且

$$
\operatorname{supp} \Psi \subset \bar{\Omega}=C .
$$

另一方面, 如果 $x \in \Omega$, 那么至少存在一个 $Q_{i}$ 满足 $Q_{i} \ni x$, 因此, $\varphi_{i}(x)>0$, 这样就可得到 $\Psi(x) \neq 0$. 结合 $(2.15)$ 立即得出

$$
\operatorname{supp} \Psi=C .
$$

最后将证明 $\Psi$ 是充分光滑的, 即需要证明 $\Psi \in C^{\infty}\left(\mathbb{R}^{n}\right)$. 为此, 分三种情形来证明这一问题.

情形 1 如果 $x \in \Omega$, 那么由分解中 $\left\{Q_{j}\right\}$ 所满足的性质 (3) 以及 $\varphi$ 所满足的三个性质可以看出, $\left\{\varphi_{j}\right\}_{j=1}^{\infty}$ 中至多存在有限个函数满足 $\varphi_{j}(x) \neq 0$. 事实上, 能证明不为零的函数的个数不超过 $12^{n}$ (参 见文献 [4]). 再次运用分解中 $\left\{Q_{j}\right\}$ 所满足的性质 (3) 以及 $\varphi$ 的三个性质可以得出, 存在一个充分小的 正数 $\delta_{x}$, 使得 $0<\delta_{x} \ll d_{x}$, 并且 $B\left(x, \delta_{x}\right) \subset \Omega$; 此外, 在球 $B\left(x, \delta_{x}\right)$ 内, $\left\{\varphi_{j}\right\}_{j=1}^{\infty}$ 中不为 0 的函数最多 为有限个, 不妨把它们记为 $\varphi_{j_{1}}, \varphi_{j_{2}}, \ldots, \varphi_{j_{m}}$. 由于这些函数在 $x$ 点处均是充分光滑的, 所以, 在 $(2.14)$ 中由 $\varphi_{j_{1}}, \varphi_{j_{2}}, \ldots, \varphi_{j_{m}}$ 构成的有限和在 $x$ 点也是充分光滑的. 这样就证明了 $\Psi$ 在 $x$ 处充分光滑.

情形 2 注意到 $C^{c}=\mathbb{R}^{n} \backslash C$ 是开集. 如果 $x \in C^{c}$, 那么必然存在一个小球 $B\left(x, r_{x}\right) \subset C^{c}$. 由 $\Psi$ 定 义可知, $\Psi$ 在小球 $B\left(x, r_{x}\right)$ 内恒为 0 . 因此, $\Psi$ 在 $x$ 处是充分光滑的.

情形 3 下面再考虑 $C$ 的边界上的情形.

令 $x \in \partial C$. 由 $\Psi$ 和 $\varphi_{j}$ 在 (2.14) 和 (2.13) 中的定义, 可以看出 $\Psi(x)=0$.

注意到当 $l_{j} \rightarrow 0$ 时, $\lambda_{j} \rightarrow \infty$. 通过 $\Psi$ 的定义, 不难得到级数

$$
\sum_{j=1}^{\infty}\left(1+\frac{1}{l_{j}}\right)^{-\lambda_{j}} \varphi_{j}(y)
$$

和

$$
\sum_{j=1}^{\infty}\left(1+\frac{1}{l_{j}}\right)^{-\lambda_{j}} \frac{\partial \varphi_{j}}{\partial x_{k}}(y)
$$

都是一致收玫的. 因此, 我们有

$$
\frac{\partial \Psi}{\partial y_{k}}(x)=\sum_{j=1}^{\infty}\left(1+\frac{1}{l_{j}}\right)^{-\lambda_{j}} \frac{\partial \varphi_{j}}{\partial y_{k}}(x) .
$$

记

$$
h=\left(0, \ldots, 0, h_{k}, 0, \ldots, 0\right) \in \mathbb{R}^{n} .
$$

不失一般性, 可以假定 $x+h \in \Omega$. 这样就有

$$
\frac{\partial \varphi_{j}}{\partial y_{k}}(x)=\lim _{h_{k} \rightarrow 0} \frac{\varphi_{j}(x+h)}{h_{k}} .
$$


对任意的 $\varphi_{j}$, 通过 $\varphi_{j}$ 的定义, 很容易得到

$$
\operatorname{supp} \varphi_{j} \subset \overline{(1+\varepsilon) Q_{j}} \subset \Omega,
$$

其中 $(1+\varepsilon) Q_{j}$ 表示中心和 $Q_{j}$ 相同、边长为 $Q_{j}$ 边长 $1+\varepsilon$ 倍的方体. 立刻可得到

$$
\frac{\partial \varphi_{j}}{\partial y_{k}}(x)=0
$$

把 (2.17) 代入 (2.16) 中, 便可得出

$$
\frac{\partial \Psi}{\partial y_{k}}(x)=0
$$

重复上述操作过程, 可以得到

$$
D^{\alpha} \Psi(x)=0,
$$

对任意的多重指标 $\alpha$ 都成立. 这样我们就证明了引理 6.

\section{3 定理的证明}

定理 1 的证明 令 $\Phi \in \mathscr{S}\left(\mathbb{R}^{n}\right)$. 由 Schwartz 函数的定义, 我们可以选取一个整数序列 $\left\{p_{k}\right\}_{k \geqslant 1}$ 满足

$$
\sup _{|\alpha| \leqslant k,|x| \geqslant 2^{p_{k}-1}}|x|^{k}\left|D^{\alpha} \Phi(x)\right| \leqslant \frac{1}{k}
$$

对任意的 $k \in \mathbb{N}$ 成立.

不妨假设这里的 $\left\{p_{k}\right\}_{k \geqslant 0}$ 是严格单调上升的, 并且, 当 $k \rightarrow \infty$ 时, $p_{k} \rightarrow \infty$. 若 $p_{k-1} \leqslant j<p_{k}$, 其 中 $k=1,2, \ldots$ 取

$$
\lambda_{j}=\sqrt{k},
$$

设 $\lambda_{j}$ 为满足 (3.2) 的序列, 我们便可以通过定义 (2.4) 的方式重新定义函数 $\Psi$. 由引理 3 可知, 对一 切 $x \in \mathbb{R}^{n}$, 有 $\Psi(x)>0$. 定义一个新函数如下:

$$
\Upsilon(x)=\frac{\Phi(x)}{\Psi(x)} .
$$

由于 $\Psi>0$, 因此这样定义的函数 $\Upsilon$ 是有意义的.

我们断言, $\frac{\Phi}{\Psi}$ 是 Schwartz 函数.

下面来估计函数 $\frac{\Phi}{\Psi}$ 的各阶偏导数. 对任意给定的一个多重指标 $\alpha \in \mathbb{N}_{0}^{n}$, 由 Newton-Leibniz 公式 可以看出

$$
\left|D^{\alpha}\left(\frac{\Phi(x)}{\Psi(x)}\right)\right| \leqslant C_{\alpha} \sum_{\beta+\gamma=\alpha}\left|D^{\beta} \Phi(x)\right|\left|D^{\gamma}\left(\frac{1}{\Psi(x)}\right)\right|,
$$

其中常数 $C_{\alpha}$ 仅依赖于维数 $n$ 和给定的 $\alpha$.

记 $|\gamma|$ 为 $\gamma$ 的阶, 通过简单地计算可知,

$$
D^{\gamma}\left(\frac{1}{\Psi(x)}\right)=\frac{\sum_{\left|\nu_{1}\right|+\left|\nu_{2}\right|+\cdots+\left|\nu_{m}\right|=|\gamma|, l_{\nu} \leqslant|\gamma|} C_{n, \nu, l_{\nu}}(\Psi(x))^{l_{\nu}} D^{\nu_{1}} \Psi(x) D^{\nu_{2}} \Psi(x) \cdots D^{\nu_{m}} \Psi(x)}{(\Psi(x))^{|\gamma|+1}},
$$


上式的常数 $C_{n, \nu, l_{\nu}}$ 只依赖于 $n$ 和 $\nu:=\left(\nu_{1}, \nu_{2}, \ldots, \nu_{m}\right)$, 其中 $\nu_{i} \in \mathbb{N}_{0}^{n}, i=1,2, \ldots, m$. 显然, (3.4) 右边 分子的求和只有有限项, 事实上, 项数由维数 $n$ 和 $\alpha$ 决定.

由 $\Psi$ 在 (2.4) 中的定义以及 $\phi$ 的性质可知, 对 $x \in \mathbb{R}^{n},(2.4)$ 右边的求和不超过三项. 注意到当 $2^{j} \leqslant|x| \leqslant 2^{j+1}$ 时, $\left|\nu_{1}\right|+\left|\nu_{2}\right|+\cdots+\left|\nu_{m}\right|=|\gamma|$, 所以, 我们有

$$
\left|\Psi^{l_{\nu}}(x) D^{\nu_{1}} \Psi(x) D^{\nu_{2}} \Psi(x) \cdots D^{\nu_{j}} \Psi(x)\right| \leqslant C_{n, \gamma} 2^{-(j-1)\left(\lambda_{j-1}+|\gamma|\right)} \sup _{|\mu| \leqslant|\gamma|}\left\|D^{\mu} \phi\right\|_{\infty} .
$$

把 (3.5) 代入 (3.4), 可得

$$
\left|D^{\gamma}\left(\frac{1}{\Psi(x)}\right)\right| \leqslant \frac{C_{n, \gamma, \varphi} 2^{-(j-1)\left(\lambda_{j-1}+|\gamma|\right)}}{|\Psi(x)||\gamma|+1},
$$

其中常数 $C_{n, \gamma, \varphi}$ 只依赖于 $n, \gamma$ 和 $\varphi$.

由引理 3 可知, 当 $x \in \mathbb{R}^{n}$ 时, $0<\Psi(x) \leqslant 1$, 并且, 当 $j>0$ 时, $2^{-(j-1)\left(\lambda_{j-1}+|\gamma|\right)} \leqslant 1$ (因为指数小 于等于 0$)$, 因此, 很容易得出

$$
\left|D^{\gamma}\left(\frac{1}{\Psi(x)}\right)\right| \leqslant \frac{C_{n, \gamma, \varphi} 2^{-(j-1)\left(\lambda_{j-1}+|\gamma|\right)}}{|\Psi(x)|^{|\alpha|+1}} \leqslant \frac{C_{n, \alpha, \varphi}}{|\Psi(x)|^{|\alpha|+1}},
$$

对任意的 $|\gamma| \leqslant|\alpha|$ 成立.

为了得到 $\frac{\Phi}{\Psi} \in \mathscr{S}\left(\mathbb{R}^{n}\right)$, 我们只需证明, 对任意给定的正整数 $N$, 极限

$$
\lim _{|x| \rightarrow \infty} \sup _{|\alpha| \leqslant N}|x|^{N}\left|D^{\alpha}\left(\frac{\Phi(x)}{\Psi(x)}\right)\right|=0
$$

成立即可.

为此, 我们仅需考虑 $|x|$ 充分大的情形, 对这样 $|x|$, 存在一个足够大的正整数 $j$ 满足: $2^{j} \leqslant|x|$ $<2^{j+1}$. 因 $j$ 充分大, 由序列 $p_{N+k}$ 的性质可知, 必然存在正整数 $k \in \mathbb{N}$, 使得 $p_{N+k} \leqslant j+1<p_{N+k+1}$. 由 (3.2) 的定义可知,

$$
\lambda_{j+1}=\sqrt{N+k+1}
$$

结合 (3.3) 和 (3.6), 我们得到

$$
\sup _{2^{j} \leqslant|x|<2^{j+1},|\alpha| \leqslant N}|x|^{N}\left|D^{\alpha}\left(\frac{\Phi(x)}{\Psi(x)}\right)\right| \leqslant \sup _{2^{j} \leqslant|x|<2^{j+1},|\alpha| \leqslant N} C_{n, N, \varphi}|x|^{N}\left(\sum_{|\beta| \leqslant|\alpha|}\left|D^{\beta} \Phi(x)\right|\right)|\Psi(x)|^{-(N+1)} .
$$

由引理 3 可知, 当 $2^{j} \leqslant|x|<2^{j+1}$ 时, 可以推出

$$
|\Psi(x)|^{-(N+1)} \leqslant 2^{(j+1) \lambda_{j+1}(N+1)} .
$$

利用 (3.9) 和 (3.10) 不难得到

$$
\sup _{2^{j} \leqslant|x|<2^{j+1},|\alpha| \leqslant N}|x|^{N}\left|D^{\alpha}\left(\frac{\Phi(x)}{\Psi(x)}\right)\right| \leqslant C_{n, N, \varphi} 2^{(j+1)(2 N+1) \lambda_{j+1}} \sup _{2^{j} \leqslant|x|<2^{j+1}}\left(\sum_{|\alpha| \leqslant N}\left|D^{\alpha} \Phi(x)\right|\right) .
$$

注意到 $p_{N+k} \leqslant j+1<p_{N+k+1}$, 结合 (3.1) 的估计, 我们有

$$
\sup _{2^{j} \leqslant|x|<2^{j+1}}\left(\sum_{|\alpha| \leqslant N}\left|D^{\alpha} \Phi(x)\right|\right) \leqslant \sum_{|\alpha| \leqslant N} 2^{-j(N+k)}\left(\sup _{2^{j} \leqslant|x|<2^{j+1}}|x|^{N+k}\left|D^{\alpha} \Phi(x)\right|\right)
$$




$$
\begin{aligned}
& \leqslant \sum_{|\alpha| \leqslant N} 2^{-j(N+k)}\left(\sup _{2^{p_{N+k}-1} \leqslant|x|<2^{j+1}}|x|^{N+k}\left|D^{\alpha} \Phi(x)\right|\right) \\
& \leqslant \sum_{|\alpha| \leqslant N} 2^{-j(N+k)}\left(\sup _{|x| \geqslant 2^{p_{N+k}-1}}|x|^{N+k}\left|D^{\alpha} \Phi(x)\right|\right) \\
& \leqslant \frac{C_{N} 2^{-j(N+k)}}{N+k},
\end{aligned}
$$

其中 $C_{N}$ 只依赖于 $N$.

由不等式 (3.11) 和 (3.12) 可以得到

$$
\sup _{2^{j} \leqslant|x|<2^{j+1},|\alpha| \leqslant N}|x|^{N}\left|D^{\alpha}\left(\frac{\Phi(x)}{\Psi(x)}\right)\right| \leqslant \frac{C_{N} C_{n, N, \varphi} 2^{-\left[j(N+k)-(j+1)(2 N+1) \lambda_{j+1}\right]}}{N+k},
$$

其中 $C_{n, \varphi, N}$ 依赖于 $n, \varphi$ 和 $N$.

注意到, 当 $j \rightarrow \infty$ 时, $k \rightarrow \infty$. 因此, 对任意给定的 $N \in \mathbb{N}$, 我们通过 (3.8) 得到

$$
j(N+k)-(j+1)(2 N+1) \lambda_{j+1} \geqslant j(k-3 N \sqrt{N+k+1}) \rightarrow \infty,
$$

上面的极限取至于 $j \rightarrow \infty$ 和 $k \rightarrow \infty$.

把 (3.14) 代入 (3.13) 可得

$$
\lim _{j \rightarrow \infty} \sup _{2^{j} \leqslant|x|<2^{j+1},|\alpha| \leqslant N}|x|^{N}\left|D^{\alpha}\left(\frac{\Phi(x)}{\Psi(x)}\right)\right|=0 .
$$

这意味着 (3.7) 成立, 即

$$
\frac{\Phi}{\Psi} \in \mathscr{S}\left(\mathbb{R}^{n}\right)
$$

这就是定理 1 所要的结论.

定理 2 的证明 定理 2 的证明思想与定理 1 的证明有许多相似之处, 在这里, 我们会省略部分 类似的细节.

令 $\Phi \in \mathscr{D}\left(\mathbb{R}^{n}\right)$. 由 (2.6), 我们可以选取一个正数序列 $\left\{p_{k}\right\}_{k \geqslant 1}$, 使得对任意的正整数 $k$ 和 $x \in \Omega$, 不等式

$$
\sup _{d_{x}<2^{-p_{k}}} d_{x}^{-k}\left(\sum_{|\alpha| \leqslant k}\left|D^{\alpha} \Phi(x)\right|\right) \leqslant \frac{1}{k}
$$

成立.

不失一般性, 可以假定 $\left\{p_{k}\right\}_{k \geqslant 1}$ 是单调上升的, 并且当 $k \rightarrow \infty$ 时, $p_{k} \rightarrow \infty$. 类似于定理 1 , 当 $2^{-p_{k+1}} \leqslant l_{j}<2^{-p_{k}}$ 时, 取

$$
\lambda_{j}=\sqrt{k+1},
$$

我们用 $(2.14)$ 定义一个函数 $\Psi$, 这里的 $\lambda_{j}$ 由 (3.17) 给出. 显然, 当 $l_{j} \rightarrow 0$ 时, $\lambda_{j} \rightarrow \infty$, 并且满足, 若 $l_{i} \approx l_{j}$, 则有 $\lambda_{i} \approx \lambda_{j}$.

定义一个新的函数

$$
\Upsilon(x)= \begin{cases}\frac{\Phi(x)}{\Psi(x)}, & x \in \Omega, \\ 0, & x \in \Omega^{c} .\end{cases}
$$


显然, $\Upsilon$ 的定义是有意义的, 因为对任意的 $x \in \Omega$, 都有 $0<\Psi(x) \leqslant 1$. 由 $\Upsilon$ 的定义我们能看出

$$
\operatorname{supp} \Upsilon=\operatorname{supp} \Phi=C .
$$

下面只需要证明 $\Upsilon \in C^{\infty}\left(\mathbb{R}^{n}\right)$ 即可.

当 $x \in \Omega$ 时, 由于 $\Phi$ 和 $\Psi$ 在 $x$ 点都是无穷次可微, 且 $0<\Psi(x) \leqslant 1$, 因此可以推出 $\Upsilon$ 在 $x$ 点是 无穷次可微的. 当 $x \in C^{c}$ 时, 显然, $\Upsilon$ 在 $x$ 点是无穷次可微的. 下面只考虑对于边界上的点, $\Upsilon$ 也是 无穷次可微的.

令 $x$ 为边界 $\partial C$ 上的一点. 由 $\Upsilon$ 在 (3.18) 中的定义, 则有 $\Upsilon(x)=0$.

记 $h=\left(0, \ldots, 0, h_{i}, 0, \ldots, 0\right) \in \mathbb{R}^{n}$, 其中 $h_{i} \neq 0$. 不失一般性, 假定 $x+h \in \Omega$. 由导数的定义可得

$$
\frac{\partial \Upsilon}{\partial x_{i}}(x)=\lim _{h_{i} \rightarrow 0} \frac{\Upsilon(x+h)}{h_{i}}=\lim _{h_{i} \rightarrow 0} \frac{1}{h_{i}} \frac{\Phi(x+h)}{\Psi(x+h)} .
$$

显然, 可以得到

$$
d_{x+h} \leqslant|x+h-x|=\left|h_{i}\right| .
$$

若

$$
2^{-p_{k+1}} \leqslant d_{x+h}<2^{-p_{k}},
$$

则根据不等式 (3.16) 和引理 6(2) 可知,

$$
\frac{1}{\left|h_{i}\right|}\left|\frac{\Phi(x+h)}{\Psi(x+h)}\right| \leqslant \frac{1}{k\left|h_{i}\right|} \frac{d_{x+h}^{k}}{d_{x+h}^{\lambda_{k+1}}} \leqslant \frac{1}{k} d_{x+h}^{k-\sqrt{k+1}-1} .
$$

显然, 当 $h_{i} \rightarrow 0$ 时, 则有 $k \rightarrow \infty$, 并且 $d_{x+h} \rightarrow 0$. 因此可以得到

$$
\frac{\partial \Upsilon}{\partial x_{i}}(x)=0 .
$$

重复上面极限过程, 对任意的多重指标 $\alpha$, 即可得到下面等式:

$$
D^{\alpha} \Upsilon(x)=0 .
$$

所以, 综合 (3.19) 和 (3.20) 就可得到

$$
\Upsilon=\frac{\Phi}{\Psi} \in \mathscr{D}\left(\mathbb{R}^{n}\right)
$$

这样就完成了定理 2 的证明.

\section{4 一些应用}

下面给出本文两个定理的直接应用.

首先定义两个映射 $\mathcal{P}$ 和 $\mathcal{C}: \mathscr{S}\left(\mathbb{R}^{n}\right) \times \mathscr{S}\left(\mathbb{R}^{n}\right) \rightarrow \mathscr{S}\left(\mathbb{R}^{n}\right)$, 分别称为乘积映射和卷积映射如下:

$$
\mathcal{P}(\varphi, \psi)=\varphi \psi
$$

和

$$
\mathcal{C}(\varphi, \psi)=\varphi * \psi
$$


定理 7 由 (4.1) 定义的乘积映射 $\mathcal{P}$ 在 $\mathscr{S}\left(\mathbb{R}^{n}\right)$ 拓扑意义下是从 $\mathscr{S}\left(\mathbb{R}^{n}\right) \times \mathscr{S}\left(\mathbb{R}^{n}\right)$ 到 $\mathscr{S}\left(\mathbb{R}^{n}\right)$ 的 一个连续满射, 但不是单射.

证明 首先来说明 $\mathcal{P}$ 是满射. 对任意的 $\Phi \in \mathscr{S}\left(\mathbb{R}^{n}\right)$, 由定理 1 立即得出存在两个 Schwartz 函数 满足 $\varphi$ 和 $\psi$, 使得

$$
\Phi=\varphi \psi
$$

这蕴含着 $\mathcal{P}$ 是满的, 即 $\mathcal{P}(\varphi, \psi)=\Phi$.

显然, $\mathcal{P}$ 不是单射. 假设 $\Phi=\varphi \psi$, 并且 $\varphi, \psi \in \mathscr{S}\left(\mathbb{R}^{n}\right)$. 进一步, 有 $2 \varphi, \psi / 2 \in \mathscr{S}\left(\mathbb{R}^{n}\right)$, 且

$$
\mathcal{P}\left(2 \varphi, \frac{\psi}{2}\right)=\Phi=\varphi \psi .
$$

这就证明了 $\mathcal{P}$ 不是单射.

下面证明 $\mathcal{P}$ 是连续的. 在 $\mathscr{S}\left(\mathbb{R}^{n}\right)$ 的拓扑意义下, 假定 $\varphi_{k} \rightarrow \varphi, \psi_{l} \rightarrow \psi$. 对多重指标 $\alpha, \beta \in \mathbb{N}_{0}^{n}$ 和 $\varphi \in \mathscr{S}\left(\mathbb{R}^{n}\right)$, 记 $\rho_{\alpha, \beta}(\varphi)=\sup _{x \in \mathbb{R}^{n}}\left|x^{\alpha} D^{\beta} \varphi(x)\right|$,

$$
\begin{aligned}
\lim _{k, l \rightarrow \infty} \rho_{\alpha, \beta}\left(\varphi_{k} \psi_{l}-\varphi \psi\right)= & \lim _{k, l \rightarrow \infty} \sup _{x \in \mathbb{R}^{n}}\left|x^{\alpha} D^{\beta}\left(\varphi_{k}(x) \psi_{l}(x)-\varphi(x) \psi(x)\right)\right| \\
\leqslant & \lim _{k, l \rightarrow \infty} \sup _{x \in \mathbb{R}^{n}}\left|x^{\alpha}\right|\left(\left|D^{\beta}\left(\varphi_{k}\left(\psi_{l}(x)-\psi(x)\right)\right)\right|+\left|D^{\beta}\left(\left(\varphi_{k}(x)-\varphi(x)\right) \psi(x)\right)\right|\right) \\
\leqslant & C_{v, \gamma} \lim _{k, l \rightarrow \infty} \sup _{x \in \mathbb{R}^{n}}\left|x^{\alpha}\right| \sum_{v+\gamma=\beta}\left|D^{v} \varphi_{k}(x)\right|\left|D^{\gamma}\left(\psi_{l}(x)-\psi(x)\right)\right| \\
& +C_{v, \gamma} \lim _{k, l \rightarrow \infty} \sup _{x \in \mathbb{R}^{n}}\left|x^{\alpha}\right| \sum_{v+\gamma=\alpha}\left|D^{v}\left(\varphi_{k}(x)-\varphi(x)\right)\right|\left|D^{\gamma} \psi(x)\right| \\
\leqslant & C_{v, \gamma} \sum_{v+\gamma=\beta} \lim _{k, l \rightarrow \infty}\left[\rho_{0, \gamma}\left(\psi_{l}-\psi\right) \rho_{\alpha, v}\left(\varphi_{k}\right)+\rho_{0, v}\left(\varphi_{k}-\varphi\right) \rho_{\alpha, \gamma}(\psi)\right] .
\end{aligned}
$$

根据假设, $\varphi_{k} \rightarrow \varphi, \psi_{l} \rightarrow \psi$, 可以立刻得出

$$
\lim _{l \rightarrow \infty} \rho_{0, \gamma}\left(\psi_{l}-\psi\right)=0, \quad \lim _{k \rightarrow \infty} \rho_{\alpha, v}\left(\varphi_{k}-\varphi\right)=0, \quad \lim _{k \rightarrow \infty} \rho_{\alpha, v}\left(\varphi_{k}\right) \leqslant C_{0, \beta}<\infty, \quad \rho_{\alpha, \gamma}(\psi) \leqslant C_{\alpha, \beta}<\infty .
$$

因此, 通过 (4.3) 可推出

$$
\lim _{k, l \rightarrow \infty} \rho_{\alpha, \beta}\left(\varphi_{k} \psi_{l}-\varphi \psi\right)=0
$$

这样就证明了定理 7 .

推论 8 在 $\mathscr{S}\left(\mathbb{R}^{n}\right)$ 的拓扑意义下, 由 $(4.2)$ 定义的卷积映射 $\mathcal{C}$ 是从 $\mathscr{S}\left(\mathbb{R}^{n}\right) \times \mathscr{S}\left(\mathbb{R}^{n}\right)$ 到 $\mathscr{S}\left(\mathbb{R}^{n}\right)$ 的连续满射, 但不是单射.

注意到 Fourier 变换是从 $\mathscr{S}\left(\mathbb{R}^{n}\right)$ 到自身的同胚映射, 由定理 7 很容易得出推论 8 .

类似地, 可以定义乘积映射 $\mathcal{Q}: \mathscr{D}\left(\mathbb{R}^{n}\right) \times \mathscr{D}\left(\mathbb{R}^{n}\right) \rightarrow \mathscr{D}\left(\mathbb{R}^{n}\right)$ 为

$$
\mathcal{Q}(\varphi, \psi)=\varphi \psi
$$

那么可以得到类似于定理 7 的结论.

推论 9 由 (4.4) 定义的乘积映射 $\mathcal{Q}$ 在 $\mathscr{D}\left(\mathbb{R}^{n}\right)$ 拓扑意义下是从 $\mathscr{D}\left(\mathbb{R}^{n}\right) \times \mathscr{D}\left(\mathbb{R}^{n}\right)$ 到 $\mathscr{D}\left(\mathbb{R}^{n}\right)$ 的连 续满射, 但它不是单射.

推论 9 的证明与定理 7 的证明方法完全相同, 只需把在 $\mathscr{S}\left(\mathbb{R}^{n}\right)$ 上的拓扑换成在 $\mathscr{D}\left(\mathbb{R}^{n}\right)$ 拓扑意 义即可. 然而, 需要指出的是, 对于卷积映射而言, 推论 8 的结论在 $\mathscr{D}\left(\mathbb{R}^{n}\right)$ 上不成立. 这是因为紧支集 函数的 Fourier 变换将不再是紧支集函数. 


\section{参考文献}

1 Kamiński A. On the convolution of distributions. In: Proc Internat Conf Generalized Funct Appl Math Phys. Moscom: Steklov Mathematical Institute, 1981, 274-282

2 Petzeltová H, Vrbová P. Factorization in the algebra of rapidly descrasing function on $\mathbb{R}^{n}$. Comment Math Univ Carolinae, 1978, 19: 489-499

3 Voigt J. Factorization in some Fréchet algebras of differentiable functions. Studia Math, 1984, 77: 333-348

4 Stein E M. Singular Integerals and Differentiability Properrities of Functions. New Jersey: Princeton University Press, 1970

5 Grafakos L. Classical and Modern Fourier Analysis. Beijing: China Machine Press Inc, 2005

6 Stein E M, Weiss G. Introduction to Fourier Analysis on Euclidean Spaces. New Jersey: Princeton University Press, 1971

7 Stein E M. Harmonic Analysis, Real Variable Methods, Orthogonality, and Osciallatory Integrals. New Jersey: Princeton University Press, 1993

\section{On the decomposition of Schwartz function and its applications}

\section{WEI MingQuan, SHI ZuoShunHua \& YAN DunYan}

Abstract In this paper, we use the constructive methods to show that every Schwartz function can be decomposed as a product of two Schwartz functions. The same is true for $\mathscr{D}\left(\mathbb{R}^{n}\right)$ function with the same compact set. As their applications, it immediately follows that $\mathscr{S}\left(\mathbb{R}^{n}\right)=\mathscr{S}\left(\mathbb{R}^{n}\right) \mathscr{S}\left(\mathbb{R}^{n}\right)$ and $\mathscr{D}\left(\mathbb{R}^{n}\right)=\mathscr{D}\left(\mathbb{R}^{n}\right) \mathscr{D}\left(\mathbb{R}^{n}\right)$. It should be pointed out that we use the adaptive idea and method to give the decompositions.

Keywords Schwartz function, $\mathscr{D}\left(\mathbb{R}^{n}\right)$ function, Fourier transform, compact support set $\operatorname{MSC}(2010) \quad 42 B 15,42 B 35$

doi: $10.1360 / N 012014-00247$ 